\title{
Third Language Acquisition With or Without the Help of English as a Language Support
}

\author{
Ana Mihaela Istrate (Lecturer, PhD) \\ Romanian-American University, Romania
}

Doi:10.19044/esj.2019.v15n5p146 ～URL:http://dx.doi.org/10.19044/esj.2019.v15n5p146

\begin{abstract}
The present study, in the field of third language acquisition, is the result of a three-year research on groups of adult students involved in learning Romanian as a foreign language for the purpose of continuing their academic studies in Romania. This paper focuses on understanding the best methods and techniques for teaching third language. This could apply to groups of international students, whose level of understanding of the English language is A1-A2. The conclusion drawn in this particular case shows that English as a language support has become a useless tool and there is therefore the need to approach the group with a full immersion method. On the other hand, we will discuss aspects related to the cultural background of the target group which dramatically affects the acquisitions of a language whose grammatical structure is extremely different from the first language. At the same time, we will focus on aspects related to the efficiency of teaching with the help of the modern communicative techniques or based on the traditional methods of teaching foreign languages. Based on an analysis of the differences between individuals coming from extremely different cultural backgrounds, we will draw comparisons between the subjects with different language performance. This is an indicator that it is directly related to their long-term objectives. The ultimate role is to raise public attention in the development and adaptation of curriculum content to student achievements, and to find a more successful approach to teaching the L3 to students with lower levels in the L2.
\end{abstract}

Keywords: Third language acquisition, Romanian as a foreign language, communicative method, English as a support language

\section{Introduction}

In today's society, due to the free movement of individuals, cheaper transportation costs and unrestricted access to international educational programs, as well as a result of the 2015 migration crisis, have led to the transfer of more than 1,300,000 people reaching Europe. According to the 
UNHCR, the preoccupation of public and non-governmental institutions was focused on the development of programs which concentrated on highlighting the concept of multilingual cultures.

In this context, the development of studies in third language acquisition (TLA) became a new topic of research which focused on a combination of "formal linguistic (Rothman, 2010), psycholinguistic (Cenoz, 2001), sociolinguistics (Bhatia \& Ritchie, 2013), and educational or applied perspectives (Cenoz, Hufesein, \& Jessner, 2001) that developed theories, frameworks, and approaches to learn and understand better how a multilingual mind works when acquiring a foreign language". (Duhalde Solis, 2015, p.2)

According to Cenoz (2003), TLA refers to the "acquisition of a nonnative language, by learners who have previously acquired or are acquiring two other languages. The acquisition of the first two languages can be simultaneous (as in early bilingualism) or consecutive." (Cenoz, 2003, p.71)

The present study represents a sociological analysis of the behavior and learning patterns of groups of international students, enrolled with an academic program for learning Romanian as a foreign language, most of whom are bilingual. They also have different cultural backgrounds and different levels in foreign language acquisition.

The first group is made up of students who were born in a different country from the origin of their parents (Algerian or Moroccan born in France, Palestinians born in Israel). Another group is made up of students from African countries which are former French colonies, and where French and a local dialect are simultaneously used by the international students. The third category includes students who are proficient in English, although their L1 is Persian or Chinese.

The final group is made up of students who are enrolled with this academic program, but Romanian is at the level of SLA.

The problem arises from the fact that at the selection level, the academic institution does not separate students according to this L2, L3 criterion. Thus, the trainers face the challenge of teaching the foreign language to a non-homogenous group of students with different learning methods. The challenge is also related to the fact that bilingual students can transfer knowledge from one language to another, make connections, draw comparisons and find similarities easier than the other students.

\section{The Importance of Cultural Background in TLA}

Particularly useful in second language acquisition is the concept of communicative competence, which arose from the criticism raised against Chomsky's notion of linguistic competence (1965). This is based on four components that are also successfully applied in the third language 
acquisition, with certain remarks and observations that need to be added to the original theory (Safont Jordà, 2005, p.51).

Canale and Swan in 1980 and later on Canale (1983) stated four important components: grammatical competence, sociolinguistic competence, strategic competence, and discourse competence that can make us understand which processes are entangled by second or third language acquisition. This also includes the aspects that learners should work on, in order to improve their performances.

Grammatical Competence refers to the acquisition of vocabulary, morphological and syntactic structures, phonology and semantics. As specialists affirm, it focuses also on learner's abilities to produce a language with a higher level of accuracy.

Sociolinguistic Competence aims at developing, in the second language learner, a set of rules and laws for apprehending and decoding the social meaning of a discourse based on the context and situation.

Strategic Competence is strongly related to an individual's ability to cope with the difficulties of communication, and his/her power to reinforce the will to communicate.

At this point, we have to admit that cultural differences play an important role in the development of strategic competences. This is because in Edward hall's terms of high context cultures vs. low context cultures, we can say that for students coming from certain parts of the world where social hierarchies are important, or gender and race differences guide the social interaction, it will be very difficult for them to find an appropriate method for mastering the strategic competences.

Discourse Competence refers to "the ability to combine linguistic forms in the production and interpretation of unified oral or written texts". (Safont Jordà, 2005, p.52)

Canale and Swain's theory was extremely successful when it appeared, although there have been voices suggesting that a detailed description would be extremely necessary.

Bachman (1990), in response to the above-mentioned theory, developed a model in which he also included the pragmatic competence as a more important tool in language acquisition. This is seen as a sort of mediator between the language and the language users.

Furthermore, when learning a foreign language, one of the most important lessons is related to advising students to be absorbed into the new culture, interact with groups of native speakers, accept the help of study buddies and be exposed to authentic language. However, as we suggested before, the cultural background of the students represents an important factor.

At the university where we teach Romanian as a Foreign Language, within the last three academic years, we have observed that the students 
enrolled with the Foundation Year of Romanian Language usually follow almost the same path. The previous years' graduates usually recommend the study programs to their younger friends, family members or colleagues back home. Thus every year they belong almost to the same cultural group.

It is also noted that, due to the recent migration wave which started in 2015, many students from the Middle Eastern countries, particularly from very sensitive war zones, were observed. Thus, we had students from Syria, Iraq, Lebanon, Israel (from the Palestinian zone), and even Iran.

There is another access route towards Europe from the African continent. Thus, we enrolled students from Algeria, Morocco, Libya, Nigeria, Burkina Faso, French Guyana, Ivory Coast, Benin or Central African Republic.

They are usually attracted by the Medical School Program, in public schools in Romania, with competitive costs in the market compared to other European countries. They are also fascinated by the fact that the diploma will offer them the opportunity to work on the free European labor market.

Thus, we can say that they belong to a group of students that are highly determined to learn the language, during the one-year program, as they are very much aware of the importance of their linguistic proficiency. In this case, we can say that there is an inner trigger that pushes the limits of the learners.

On the other hand, if we cope with students whose only purpose was to leave the conflict zones from their home countries, but do not yet have clear ideas about the future career paths that they would like to follow in life, then the whole teaching-learning process is hampered. This is not only because of the linguistic barrier, but also by their resistance to the new culture that they sometimes reject or refuse to adapt to.

\section{Methods and Techniques Used in Teaching the $\mathbf{L 3}$}

When we talk about third language acquisition, we have to admit that the process is not exactly the same as in the second language acquisition, as it means an augmentation of the linguistic system of the learner, both from a qualitative and quantitative point of view. This is why, in many situations, it seems as a more difficult and complex process.

Furthermore, we should also add the roots of the languages. If, for example, we have an Arabic speaking learner that has a pre-intermediate to intermediate level in English, the acquisition of Romanian which is also part of the Romanian languages family can seem as an almost impossible task.

On the other hand, if we have a bilingual student from a French speaking country, in North Africa that has an upper intermediate level in English, he/she will be able to create a system of connections which is based on similarities between languages, common grammatical structures, and common syntax that will dramatically ease the process. 
Researchers suggest that "one of the principal predictors of source(s) of interlanguage transfer in the L3 learner is language typology (Cenoz, 2003) or even psychotypology (Kellerman, 1983; Ringbom, 2001). This is in relation with transfer of word from being more prevalent between languages that are typologically similar in relation to those that are not, regardless of order of acquisition." (Foote, 2009, p.89)

The question that appears is related to how much of the information from the L1 or L2 is transferred into L3. Field search and case studies by Foote in 2009 suggest that the more trained the learner is in L2, the more efficient and effective the acquisition of L3 language is, particularly in terms of morphology and syntax.

Peal and Lambert (1962) suggest that bilingual students have a cognitive advantage, although this advantage is not achieved until they reach a certain level of proficiency. For example, in the case of a student, born in France from Algerian parents who enrolled with the Foundation Year of Romanian Language, her performance was dramatically increased by her mastering of two different linguistic systems. This showed the L3 having the same Latin root as her L2 language, French.

The research and analysis show that the bilingual proficiency in both languages seems to have an advantage over monolinguals when acquiring the L3 (Cenoz \&Valencia, 1994; Lasagabaster, 2000). We have also seen that a similar advantage is found for the L3 acquisition of specific features, whether or not the features are also present in the L2 (Flynn et al., 2004; Leung, 2005; Klein, 1995; Jaensch, 2009, p.124)

Learners also develop a metalinguistic experience, have an improved vocabulary, and can easily make acoustic connections between words that sound similar in L2 and L3 or L1 and L3. In other words they have a higher cognitive competence.

In 2002, Ringbom developed a theory for an understanding of psychotypological transfer of language, based on form and meaning, suggesting that there are three levels of transfer which he calls: overall level, item level, and system level.

The overall level, as the name suggests, refers to the learner's overall perception upon "beginning from a common alphabet and phonemes in common over the division into grammatical categories (case, gender, word classes) to the number of cognates and other lexical similarities" (Ringbom, 2002, p.1)

Item transfer, as stated by Ringbom, refers to the interlingual identifications that are performed during the new language acquisition.

System transfer refers to the identification, by the learners, "of an identity of meaning between items, but not necessarily of form". (De Angelis, 2007, p.25) 
If we refer to the motivation for learning the L3, and we do not consider the bilingual aspect, we have to split the groups of learners into two separate groups:long term oriented and informal learners.

- The long-term oriented (Dina, 2013) are those who wish to develop a career in an academic institution and need the language for this purpose.

- The informal learners, who are simply driven by their families, do not know yet the career paths they would like to follow. There could also be different reasons behind the application for an L3 program.

Therefore, this is why the groups of international students are not very homogenous, and there is an academia need to tackle differently these groups of students.

It is significantly important to take into cognizance the necessary process of separation, as this is based on their future program (medical school, business or technical school). This is because the specialized language classes need to be dealt with separately.

Furthermore, we could observe large variations in their understanding of L2, within the three years of our research when we took different approaches. During the first year, we made use of English as the language support, and this method was also determined by the teaching material used, which contained detailed grammatical explanation in English.

The method proved its efficiency mostly with students from Arabian countries, compared to the Chinese or French speaking African students. If the Chinese successfully adopted the study buddies and were able to improve their skills and keep the pace with the rest of the group, the method would seem more difficult in the case of French speaking students. However, due to the similarities in the grammatical and lexical structure of the two languages, they showed an efficient acquisition of the L3.

According to a study by Dina, she refers to the young ones as homogenous groups of learners (17 to 30-year-old segment), while nonhomogenous groups include the adult learners (30 to 40 years-old learners).

Indeed, it becomes a problem when teaching young and adult learners together, because the pedagogical approach needs to be adjusted to the pace of each category. On the other hand, students have the tendency of splitting in the class according to this criterion.

During the second year of our analysis, with a new group of students sharing almost the same demographic criteria, we adjusted the teaching methodology and upon the recommendations of the specialists in third language teaching, we switched to teaching directly in L3 using the natural approach.

Thus, this method generally improves the development of the communicative skills, help them to guess and recognize meaning, develop 
strategies for creating connections, and transfer the L3 learners from the preproduction stage to the early production stage.

During the last year of our study, we performed an initial separation of the international students into two different groups. This comprised of those learning Romanian as L2 in a group and L3 in another group. According to a holistic view of bilingualism, specialists consider that a third language acquisition means a widening of the linguistic system. Thus, learners involved in the process have different needs and usually ask different questions from the students involved in the second language learning.

There are different cognitive mechanisms developed by L2 and L3 learners, and we were able to observe a faster pace of the teaching-learning process in the L3 group than in the L2. This is due to the fact that L3 learners have in mind the grammatical structure, the theoretical notions of syntax, develop adaptive learning processes and probably have a different degree of awareness in the process of language acquisition.

\section{Conclusion}

Studying the patterns for the acquisition of foreign languages has always been of great interest for the specialists in the field of applied linguistics.

However, the phenomenon of multilingualism became of great interest in the last twenty years. This was when the differentiation between second language acquisition and third language acquisition became very clear and was supported by the different theoretical approaches: educational (Cenoz, 2003), neurolinguistics (Bardel \& Falk, 2013) or formal linguistic (Garcia Mayo, 2012).

One of the important conclusions that can be drawn refers to the fact that there are different factors involved in L2 or L3 acquisitions. There are also special inner triggers that assist individuals to start learning a new language at adult level (e.g. professional or personal development, career path, etc).

At the same time, we can identify different factors that determine the speed with which learners will reach the proficiency in the language level.

Research in third language acquisition also shows that the transfer can be made directly from the native language or from the second language.

However, from a methodological perspective, studies need to be deepened and what is more important is an adaptation of the teaching methods and methodologies need to be made. We need to improve our methods so that the new technological developments could be implemented into the practical seminars (e.g. beginning with 2018, Google has announced an implementation of 26 different languages in their Google Home device).

The most recent devices, such as Google Home, Alexa, Amazon Echo, etc., can be successfully used in class during the practical seminars, where the L1 to L2/L3 transfer of meaning is necessary. 
Nevertheless, we must admit that computer assisted language learning is still incipient. This is because it is difficult to train language teachers to give up their pedagogical skills in favor of smart devices. This new trend opens the window towards a new era of teaching and learning, which will replace modern day traditional methods.

\section{References:}

1. Cenoz, J. (2003). The successive effect of bilingualism on third language acquisition. A review. International Journal of Bilingualism, 7.

2. De Angelis, G. (2007). Third or Additional Language Acquisition, Second Language Acquisition 24, Multilingual Matters Ltd.

3. Dina, A.T. (2013). Successful approach for teaching Romanian as a foreign language, Procedia-Social and Behavioral Sciences 70 (2013)

4. Duhalde Solis, J.P. (2015). Third Language Acquisition: Cross Linguistic Influence form L1 and L2, Universitat Autonoma de Barcelona.

5. Falk, Y., Lindqvist, C., Bardel, C. (2013). The role of L1 explicit metalinguistic knowledge in L3 oral production at the initial state, Bilingualism Language and Cognition 18 (2), 2015

6. Foote, R. (2009). in Third Language Acquisition and Universal Grammar, edited by Yan-kit Ingrid Leung, Multilingual Matters, Bristol-Buffalo-Toronto.

7. García-Mayo, M. (2012). Cognitive approaches to L3 acquisition, in International Journal of English Studies, University of Murcia

8. Jaensch, C. (2009). in Third Language Acquisition and Universal Grammar, edited by Yan-kit Ingrid Leung, Multilingual Matters, Bristol-Buffalo-Toronto.

9. Leung, Y.I. (2009). Third Language Acquisition and Universal Grammar, Second Language Acquisition

10. Ringbom, H. (2002). Levels of transfer from L1 and L2 in L3acquisition. In J. Ytsma and M. Hooghiemstra (eds) Proceedings of the Second International Conference on Trilingualism. Leeuwaarden: Fryske Akademie.

11. Safont Jordà, M.P. (2005). Third Language Learners: Pragmatic Production and Awareness, Multilingual Matters Ltd. 